\title{
Adherence to Metformin, Statins, and ACE/ARBs Within the Diabetes Health Plan (DHP)
}

\author{
O. Kenrik Duru, MD, MSHS ${ }^{7}$, Norman Turk, $M S^{7}$, Susan L. Ettner, PhD ${ }^{1,2}$, Romain Neugebauer, PhD ${ }^{3}$, \\ Tannaz Moin, MD, MBA, MSHS ${ }^{4,5}$, Jinnan $\mathrm{Li}, \mathrm{MPH}^{7}$, Lindsay Kimbro, MPP ${ }^{7}$, Charles Chan, MS ${ }^{6}$, \\ Robert H. Luchs, MS $S^{6}$, Abigail M. Keckhafer, MBA, MPH', Anya Kirvan, RN, MS 6 , Sam Ho, MD ${ }^{6}$, and \\ Carol M. Mangione, MD, MSPH ${ }^{1,2}$
}

\begin{abstract}
'David Geffen School of Medicine at UCLA, University of California, Los Angeles, CA, USA; ${ }^{2}$ Fielding School of Public Health, University of California,

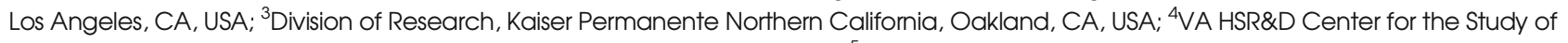
Healthcare Innovation, Implementation, and Policy, Los Angeles, CA, USA; ${ }^{5}$ VA Greater Los Angeles Healthcare System, Los Angeles, CA, USA; ¿UnitedHealthcare, Minnetonka, MN, USA.
\end{abstract}

\begin{abstract}
BACKGROUND: Reducing patient cost-sharing and engaging patients in disease management activities have been shown to increase uptake of evidence-based care.

OBJECTIVE: To evaluate the effect of employer purchase of a disease-specific plan with reduced cost-sharing and disease management (the Diabetes Health Plan/DHP) on medication adherence among eligible employees and dependents.
\end{abstract}

DESIGN: Employer-level "intent to treat" cohort study, including data from eligible employees and their dependents with diabetes, regardless of whether they were enrolled in the DHP.

SETTING: Employers that contracted with a large national health plan administrator in 2009, 2010, and/or 2011. PARTICIPANTS: Ten employers that purchased the DHP and 191 employers that did not (controls). Inverse probability weighting (IPW) estimation was used to adjust for inter-group differences.

INTERVENTION: The DHP includes free or low-cost medications and physician visits. Enrollment strategies and specific benefit designs are determined by the employer and vary in practice. DHP participants are notified up front that they must engage in their own health care (e.g., receiving diabetes-related screening) in order to remain enrolled.

MAIN OUTCOME MEASURE: Mean employee adherence to metformin, statins, and ACE/ARBs at the employer level at one year post-DHP implementation, as measured by the proportion of days covered (PDC).

RESULTS: Baseline adherence to the three medications was similar across DHP and control employers, ranging from 64 to $69 \%$. In the first year after DHP implementation, predicted employer-level adherence for metformin (+4.9 percentage points, $\mathrm{p}=0.017)$, statins $(+4.8, \mathrm{p}=$ 0.019), and ACE/ARBs $(+4.4, p=0.02)$ was higher with DHP purchase.

$\overline{\text { Electronic supplementary material The online version of this article }}$ (doi:10.1007/s11606-015-3284-8) contains supplementary material, which is available to authorized users.

Received October 3, 2014

Revised February 24, 2015

Accepted March 4, 2015

Published online May 6, 2015
LIMITATIONS: Non-randomized, observational study. CONCLUSIONS: The Diabetes Health Plan, an innovative health plan that combines reduced cost-sharing and disease management with an up-front requirement of enrollee participation in his or her own health care, is associated with a modest improvement in medication adherence at 12 months.

J Gen Intern Med 30(11): 1645-50

DOI: $10.1007 / \mathrm{s} 11606-015-3284-8$

(c) Society of General Internal Medicine 2015

$\mathrm{T}$ ype 2 diabetes is highly prevalent in the United States, and leads to reduced functional status and disabling complications for many patients. The total economic cost of diabetes in 2012 has been estimated at $\$ 245$ billion, which is an increase of $41 \%$ since 2007. ${ }^{1}$ This cost includes $\$ 176$ billion in direct medical care, as well as $\$ 69$ billion in decreased productivity such as absenteeism, reduced work performance, and reduced labor force participation. ${ }^{1}$ Importantly, greater adherence to diabetes-related medications has been shown to decrease hospitalizations and emergency department use and to reduce costs of care. $^{2-4}$ Given these issues, employers have a strong interest in trying alternative approaches to providing medical care for their employees with diabetes.

The Diabetes Health Plan (DHP) is an example of a novel health benefit design that became available to public and private employers in 2009. The DHP is the first disease-specific health plan in the United States for patients with diabetes and pre-diabetes, and offers features such as reduced cost-sharing for medications and office visits and free or low-cost resources for disease management. The DHP was actuarially designed to provide an estimated annual out-of-pocket savings of \$150-\$500 per participant. $^{5}$

As some employers purchased the DHP, while other similar employers did not, there is a unique opportunity to conduct a rigorous evaluation of a real-world intervention. In the current 
study, we examined the impact of the DHP on adherence to three evidence-based medications covered by the DHP (metformin, statins, ACE/ARBs) over 12 months. We also examined adherence to two unrelated medications that were not covered by the DHP (thyroxine, montelukast) in order to test whether our findings were related to the DHP. We chose to conduct employer-level analyses in order to provide information to guide the critical decision faced by employers in terms of which health plans to offer to their employees. With the employer as the unit of analysis, we predicted the average medication adherence across all DHP-eligible employees and dependents with diabetes whose employers had purchased the DHP, regardless of whether these patients actually enrolled in the plan.

\section{METHODS}

This study was conducted under the oversight of the Natural Experiments in Translation for Diabetes (NEXT-D), a multicenter research network supported by the Centers for Disease Control and Prevention (CDC) and the National Institute of Diabetes and Digestive and Kidney Diseases (NIDDK). ${ }^{6}$ Key elements of all NEXT-D studies include the stipulation that health policies and interventions under evaluation are being implemented "naturally," without randomization, but that the analyses use the strongest research design possible in order to provide actionable evidence for private and public health policy decision-makers. The NEXT-D network strongly encourages close collaboration with the organizations implementing the programs under study in order to ensure that the research questions are relevant and timely to realworld issues. However, the academic investigators in NEXT$D$ retain sole authority over the implementation of all analyses, report writing, and publication-related decisions. For this manuscript, UnitedHealthcare coauthors provided administrative claims data and helped interpret data analyses run by the academic team. The current study was approved by the institutional review board of the University of California, Los Angeles, as well as an executive review committee within NEXT-D.

\section{Setting}

The DHP was developed by UnitedHealthcare with a standard protocol and benefit design covering antiglycemic, antihypertensive, and statin medications. ${ }^{5}$ Since 2009 , a variety of medium-sized and large employers, both public and private, have purchased and implemented the DHP. These employers have had the option of making changes to the enrollment strategy. Some employers used an "opt-in" approach, while others automatically enrolled all eligible participants ("optout"). Rates of DHP uptake among eligible employees varied widely, from a low of $8.3 \%$ for one opt-in program to a high of $85.5 \%$ for one opt-out program, with an overall mean of $45.3 \%$ and a median of $34.1 \%{ }^{7}$
Employers were also able to modify the standard benefit design in order to fit their specific needs, such that actual DHP cost-sharing varies by employer. However, the majority of DHP employers provide all covered medications at no cost or provide Tier 1 (generic) medications for free and discount the copayment on Tier 2 (formulary brand name) medications by $50 \%$. Mean copayments for three commonly prescribed medications (metformin [Glucophage], olmesartan [Benicar], and rosuvastatin [Crestor]) in the pre-period and post-period are shown in Table 1 for DHP and control employers. Most DHP employers also reduced the copayments for visits to primary care providers or endocrinologists. The DHP also includes access to online or telephone wellness coaching. Of note, eligible employees and dependents are notified up front that DHP enrollees are expected to participate in their own care with regular health risk assessments, diabetes-related screenings and other preventive care such as age-appropriate cancer screening. DHP enrollees who do not meet these process guidelines, or "compliance criteria," as set by each employer may be returned to their traditional health plan after one year. Outcome-based criteria are never required in order to maintain DHP enrollment.

\section{Study Design/Participants}

For the current analyses, we used a pre-post quasiexperimental design at the employer level, with controls, comparing employers that purchased the DHP with control employers that did not. Although 19 employers had implemented the DHP by January 1, 2011, we limited the analytic sample to ten (Fig. 1), excluding those that did not have available pharmacy claims (i.e., had external pharmacy contracts, $n=4$ ), those without at least 1 year of baseline pre-DHP data $(n=2)$ and those with missing or incomplete beneficiary enrollment data or claims $(n=3)$. Of the more than 2,000 employers who purchased standard plans, 1,388 had available pharmacy claims and were in similar industries and of sizes similar to employers who had purchased the DHP. In order to identify comparable control employers, we conducted an employer-level propensity score match on employer size, mean income, proportion of female employees, proportion of employees with a chronic condition, and an estimated measure of the overall generosity of benefits for each health plan. Propensity score matching yielded 339 employers in the common support to serve as potential controls. Among these, only 233 had sufficient administrative and laboratory data to identify employees with diabetes. We then excluded employers that had external pharmacy contracts $(n=11)$, those in which $>$ $90 \%$ of members had high-deductible health plans (defined in Publication 969 of the Internal Revenue Service, ${ }^{8} n=7$ ), and those in the Mid-Atlantic region $(\mathrm{n}=24)$, where no DHP plans were located. This resulted in a final analytic sample of 191 control employers (Fig. 1). The DHP was implemented on a rolling basis between 2009 and 2011. For DHP employers, we defined a "pre-period" and "post-period" for data collection as 
Table 1 Mean Employer-Level Copayments for 30-Day Supplies of Metformin (Glucophage), Olmesartan (Benicar) and Rosuvastatin (Crestor) for DHP-Eligible Employees and Dependents of Employers who Purchased the DHP $(n=10)$ and Control Employers $(n=201)$

\begin{tabular}{|c|c|c|c|c|c|}
\hline \multirow[t]{2}{*}{ Medication } & \multirow[t]{2}{*}{ Dosage } & \multicolumn{2}{|l|}{ DHP employers } & \multicolumn{2}{|c|}{ Control employers } \\
\hline & & $\begin{array}{l}\text { Copayment in } \\
\text { pre-period }\end{array}$ & $\begin{array}{l}\text { Copayment in } \\
\text { post-period }\end{array}$ & $\begin{array}{l}\text { Copayment in } \\
\text { pre-period }\end{array}$ & $\begin{array}{l}\text { Copayment in } \\
\text { post-period }\end{array}$ \\
\hline \multirow[t]{3}{*}{ Metformin } & $500 \mathrm{mg}$ & $\$ 7.00$ & $\$ 3.68$ & $\$ 6.26$ & $\$ 5.70$ \\
\hline & $850 \mathrm{mg}$ & $\$ 5.79$ & $\$ 3.55$ & $\$ 5.89$ & $\$ 5.45$ \\
\hline & $1,000 \mathrm{mg}$ & $\$ 7.37$ & $\$ 4.62$ & $\$ 6.57$ & $\$ 6.19$ \\
\hline \multirow[t]{3}{*}{ Olmesartan } & $5 \mathrm{mg}$ & $\$ 15.10$ & $\$ 10.00$ & $\$ 23.12$ & $\$ 22.93$ \\
\hline & $20 \mathrm{mg}$ & $\$ 23.95$ & $\$ 14.82$ & $\$ 21.32$ & $\$ 21.80$ \\
\hline & $40 \mathrm{mg}$ & $\$ 22.28$ & $\$ 15.09$ & $\$ 22.22$ & $\$ 22.67$ \\
\hline \multirow[t]{3}{*}{ Rosuvastatin } & $10 \mathrm{mg}$ & $\$ 22.66$ & $\$ 15.66$ & $\$ 21.87$ & $\$ 22.24$ \\
\hline & $20 \mathrm{mg}$ & $\$ 21.52$ & $\$ 14.17$ & $\$ 21.28$ & $\$ 21.09$ \\
\hline & $40 \mathrm{mg}$ & $\$ 20.71$ & $\$ 14.30$ & $\$ 21.36$ & $\$ 21.30$ \\
\hline
\end{tabular}

a DHP Employers

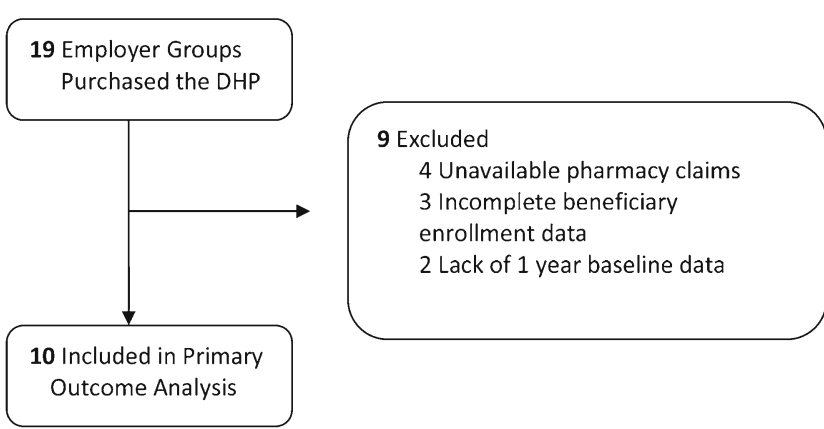

b Employers that Purchased Standard Medical Plans (i.e., controls)

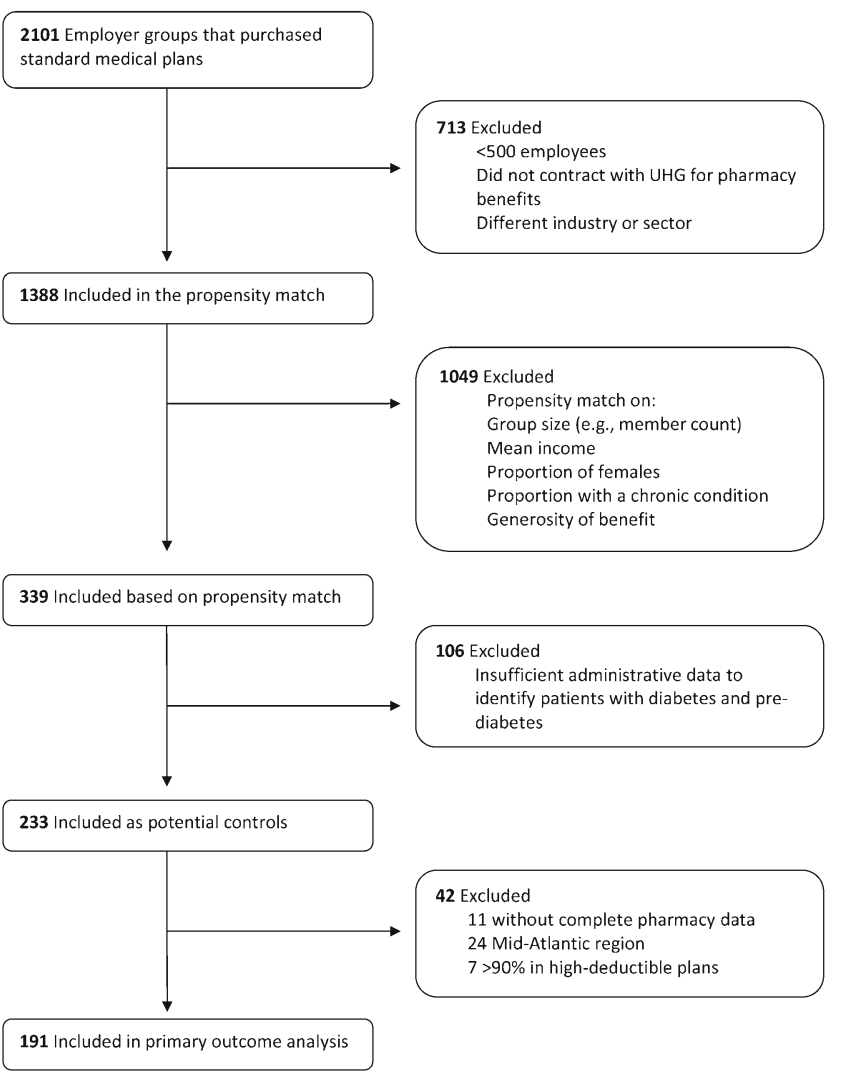

Fig. 1 Sample derivation for Diabetes Health Plan (DHP) employers and control employers. the 12 months prior to and after DHP implementation, respectively. For control employers, we defined the pre-period as 2010 and the post-period as 2011 , to match the implementation date for employers who purchased the DHP.

In order to identify eligible patients with diabetes, we applied identical criteria to DHP and control employers. Employees or dependents were considered as having diabetes if they had any of the following during the pre-period: 1) at least one 250.xx ICD-9 diagnosis code from an inpatient, outpatient, or emergency department claim; 2) laboratory values of a hemoglobin A1c $\geq 6.5 \%$, a fasting plasma glucose $\geq 125 \mathrm{mg} /$ $\mathrm{dl}$, or a 2-hour value on an oral glucose tolerance test of $>$ $200 \mathrm{mg} / \mathrm{dl}$; 3) one or more prescription fills for insulin, or 4) one or more prescription fills for an antiglycemic medication other than metformin. We limited the sample of eligible individuals to employees and dependents between the ages of 18 and 63 years on the first day of the pre-period in order to exclude patients who aged into Medicare. We excluded Medicare patients who were less than 65 years of age during the entire study window, and we excluded women who were pregnant during the study window. We analyzed outcomes from all eligible patients who were prevalent users as measured in the first quarter of the post-period, including patients from DHP employers who were not actually enrolled in the DHP.

\section{Measurement/Variables}

The primary outcome variables for these analyses were employer-level adherence in the post-period for each of five medications: metformin, statins, and ACE/ARBs (covered by the DHP); and thyroxine and montelukast (medications for conditions unrelated to diabetes and that are not covered by the DHP, included as a "falsification test"). ${ }^{9}$ Adherence was calculated as the mean proportion of days covered (PDC) over the last 9 months of the post-period, accounting for medication carry-forward from the first quarter. We did not control for fills for multiple prescriptions within a drug class (e.g., prescriptions for both an ACE and an ARB), although if two or more prescriptions were filled on the same day, we included only the prescription with the higher days' supply in the adherence calculation. Patients who filled a prescription for insulin at 
any time during the post-period were excluded from the analyses predicting adherence to metformin.

\section{Statistical Analyses}

Because of the possibility of selection bias at the employer level, we used inverse probability weighting (IPW) to adjust for differences between the two comparison groups. IPW allows for better confounding adjustment in observational studies. ${ }^{10},{ }^{11}$ First, pre-period data from eligible patients with diabetes were aggregated at the employer level to define the confounder variables used to estimate propensity scores. We then estimated propensity scores for all 201 employers, specifically including variables that we hypothesized might influence whether a given employer would purchase the DHP (e.g., employer health care costs differ by region). We included the following aggregate variables in a logistic model for the propensity score (i.e., variables were defined at the employer level by summarizing all patient data measured during the preperiod): race/ethnicity (percentage white, Hispanic, black, Asian, or other race), average salary, geographic region, number of employees, percentage of employees with an HDHP, percentage of employees with each of 15 comorbidities (hypertension, coronary artery disease, congestive heart failure, dementia, schizophrenia, another mental health condition, osteoarthritis, rheumatoid arthritis, non-skin cancer, chronic obstructive pulmonary disease, atrial fibrillation, end-stage renal disease, stroke, peripheral vascular disease, diabetes, or hyperlipidemia), and an estimated measure of the overall generosity of benefits for each health plan. We also included three additional aggregate variables specific to patients with diabetes: mean age, percentage who were female, and baseline employer-level medication adherence, defined as the average PDC in the last 9 months of the pre-period among eligible patients who were prevalent users of the drug in the first quarter of the pre-period.

We then assigned each employer a "weight" or influence that was inversely proportional to the probability of each employer being in the treatment group in which they were actually included. This approach, with the inverse propensity scores as weights, was used to estimate the average treatment effect of the treated (ATET). The ATET reflects the adjusted difference among DHP employers in the mean rates of adherence associated with purchase of the DHP compared to mean rates of adherence if those employers had not purchased the DHP. Analyses were conducted using SAS 9.3 and the "teffects" command of Stata 13.

\section{RESULTS}

The analytic sample included 201 employers, of whom ten had purchased the DHP and 191 had not. The employer groups that had purchased the DHP included 3,152 employees or covered dependents with diabetes, while those who had not purchased the DHP included 30,542 employees or covered dependents with diabetes. Unadjusted demographics were similar across groups, although control employers had a higher mean salary $(\$ 71,770$ vs. $\$ 64,782, p=0.004)$ and a higher mean percentage of Asian employees (3.9\% vs. $2.1 \%, p=$ 0.002) compared to DHP employers (Table 2). Unadjusted medication use and baseline medication adherence as measured by PDC were similar across groups. The inverse

Table 2 Unadjusted Baseline Characteristics of Diabetes Health Plan (DHP) and Control Employers (n=201)

\begin{tabular}{|c|c|c|c|}
\hline Employer-level characteristic & DHP employers $(n=10)$ & Control employers $(n=191)$ & P value \\
\hline \multicolumn{4}{|l|}{ Employer demographics } \\
\hline Mean employee salary (SD) & $\$ 64,782(5,634)$ & $\$ 71,770(16,733)$ & 0.004 \\
\hline Mean number of employees (SD) & $9,505(9,748)$ & $8,021(16,428)$ & 0.778 \\
\hline Mean employee age (SD) & $53.7(6.4)$ & $52.9(4.9)$ & 0.610 \\
\hline Mean $\%$ female (SD) & $40.7(7.0)$ & $44.9(8.6)$ & 0.129 \\
\hline Mean $\%$ of employees with diabetes (SD) & $5.4(3.1)$ & $4.3(2.0)$ & 0.308 \\
\hline \multicolumn{4}{|l|}{ Race/ethnicity distribution of employees } \\
\hline Mean \% white (SD) & $62.1(13.4)$ & $66.3(11.9)$ & 0.285 \\
\hline Mean \% black (SD) & $9.8(11.8)$ & $8.5(7.2)$ & 0.725 \\
\hline Mean \% Asian (SD) & $2.1(1.3)$ & $3.9(5.4)$ & 0.002 \\
\hline Mean \% Latino (SD) & $16.1(13.8)$ & $11.8(9.8)$ & 0.192 \\
\hline Mean $\%$ other Race (SD) & $3.3(2.6)$ & $3.0(1.4)$ & 0.648 \\
\hline \multicolumn{4}{|c|}{ Medication use among employees with diabetes, by employer } \\
\hline Mean $\%$ taking metformin (SD) & $34.8(4.9)$ & $32.8(7.8)$ & 0.443 \\
\hline Mean $\%$ taking a statin (SD) & $37.1(8.8)$ & $35.2(9.0)$ & 0.516 \\
\hline Mean $\%$ taking an ACE or ARB (SD) & $40.1(7.6)$ & $37.7(8.7)$ & 0.384 \\
\hline Mean \% taking thyroxine (SD) & $8.2(2.8)$ & $8.3(3.2)$ & 0.885 \\
\hline Mean \% taking montelukast (SD) & $1.7(0.7)$ & $1.9(1.2)$ & 0.603 \\
\hline \multicolumn{4}{|l|}{ Baseline medication adherence, by employer* } \\
\hline Mean metformin adherence in the pre-period (SD) & $64.1(7.81)$ & $67.5(7.5)$ & 0.162 \\
\hline Mean statin adherence in the pre-period (SD) & $69.4(6.6)$ & $69.4(6.8)$ & 0.999 \\
\hline Mean ACE/ARB adherence in the pre-period (SD) & $67.8(6.0)$ & $67.0(6.2)$ & 0.700 \\
\hline Mean thyroxine adherence in the pre-period (SD) & $74.3(8.6)$ & $77.8(11.1)$ & 0.334 \\
\hline Mean montelukast adherence in the pre-period (SD) $\dagger$ & $62.6(17.5)$ & $63.2(22.7)$ & 0.932 \\
\hline
\end{tabular}

* Baseline adherence was calculated as the mean proportion of days covered (PDC) over the last 9 months of the 12-month pre-period window, accounting for medication carry-forward from the first quarter

tWe found negligible use of montelukast within 20 of the control employers, and these employers were dropped from all analyses of adherence to montelukast, leaving 171 controls 
probability weights for the ten DHP employers had a mean of 1. The inverse probability weights for the 191 control employers had a mean of 0.05 and a standard deviation of 0.07 . After weighting, there were no statistically significant differences in demographic characteristics between DHP and control employees who were taking one of the five medications under study (Appendix 1).

In adjusted results (Table 3), the mean predicted adherence rates for the entire employer were greater with DHP purchase for metformin $(+4.9$ percentage points compared with no DHP purchase), statins (+4.8), and ACE/ARBs (+4.4). Each of the three comparisons was statistically significant. In contrast, we did not observe a significant change in predicted adherence to thyroxine or montelukast, medications that are not covered by the DHP.

\section{DISCUSSION}

In summary, we observed an association between employers offering the DHP and higher adherence to evidence-based medications used in diabetes treatment, as compared with employers not offering the DHP. This employer-level finding applies to all eligible employees, regardless of whether they were actually enrolled in the DHP. The lack of an observed association for medications not covered by the DHP enhances the internal validity of our results. Future studies of the DHP will use similar quasi-experimental designs to evaluate other important outcomes such as the use of wellness programs, overall utilization, and health care costs of the plan.

While our results indicated an improvement in medication adherence at 1 year post-implementation, the magnitude of change was modest. However, similar improvements in adherence in other settings have been significantly associated with decreased utilization and reduced costs. ${ }^{2}$ The DHP "benefits package" includes discounted copayments for evidencebased medications, which is similar to value-based insurance design, or VBID. Prior studies of VBID programs have shown that adherence may continue to increase at years 2 and 3 postimplementation. ${ }^{12},{ }^{13}$ Given the difficulties of communicating DHP information to enrollees, and the subsequent lag in employee engagement and participation, we expect that follow-up studies may continue to show improvements in medication adherence over time.

The RAND Workplace Wellness Study, published in 2013 after the DHP had been implemented, identifies effective implementation strategies that may be useful in the "next generation" of the DHP and similar programs. ${ }^{14}$ These strategies, which should help increase program uptake and engagement, include 1) the use of effective and clear communication strategies, 2) leadership engagement at all levels, including direct supervisors in addition to top executives, and 3) active solicitation of employee feedback, with the goal of continuous program improvement. While employers are able to modify many aspects of DHP implementation, ensuring the use of proven strategies such as those in the RAND study may result in a more robust program effect that is consistent across employers.

Demonstration of return on investment (ROI) in metrics that are highly relevant to employers will eventually be required if programs such as the DHP are to continue and expand. Such an analysis would compare the employer cost of a program with the savings in healthcare costs due to program effects (e.g., increased adherence). Because the charge to employers for administration of the DHP is proprietary, we are unable to provide it in this paper. However, a recent meta-analysis of other employer wellness programs reported an average annual cost of $\$ 144$ per employee. ${ }^{15}$ The literature on program-related cost savings indicates that a $5 \%$ increase in medication adherence, as we observed in this study, results in a $1 \%$ reduction in spending on health care services for Medicare patients, ${ }^{16}$ and a $0.6 \%$ reduction in hospitalizations and emergency department visits within a commercially insured population with diabetes. ${ }^{2}$

A complete ROI analysis is beyond the scope of this paper, but the ultimate benefit of the DHP will greatly depend on the baseline health status of the employees with diabetes and their health care utilization. Furthermore, additional studies that broaden this area of study to examine the use of incentives for leveraging patient engagement and to evaluate outcomes over a time frame of multiple years, including outcomes such as decreased absenteeism and a reduction in short-term disability, ${ }^{17},{ }^{18}$, have the potential to demonstrate the ROI of such programs in a way that resonates with the business community.

Our study has several limitations. First, we chose to conduct an employer-level analysis in order to mitigate selection bias at

Table 3 Predicted Medication Adherence at the Employer Level, With and Without Exposure to the Diabetes Health Plan (DHP)

\begin{tabular}{|c|c|c|c|c|c|}
\hline & $\begin{array}{l}\text { Predicted adherence } \\
\text { without DHP exposure }\end{array}$ & $\begin{array}{l}\text { Predicted adherence } \\
\text { with DHP exposure }\end{array}$ & Absolute difference & $\begin{array}{l}\text { Relative } \\
\text { difference }\end{array}$ & $P$ value \\
\hline Metformin & $63.1 \%$ & $67.2 \%$ & +4.1 percentage points $(95 \%$ CI: $0.7,7.5)$ & $6.5 \%$ increase & 0.017 \\
\hline Statin & $66.5 \%$ & $71.4 \%$ & +4.9 percentage points $(95 \%$ CI: $1.1,8.8)$ & $7.4 \%$ increase & 0.012 \\
\hline $\mathrm{ACE} / \mathrm{ARBs}$ & $67.1 \%$ & $71.8 \%$ & +4.7 percentage points $(95 \%$ CI: $0.7,8.6)$ & $7.0 \%$ increase & 0.02 \\
\hline Thyroxine & $70.9 \%$ & $74.0 \%$ & +3.1 percentage points $(95 \% \mathrm{CI}:-3.7,9.9)$ & $4.4 \%$ increase & 0.37 \\
\hline Montelukast & $71.4 \%$ & $67.4 \%$ & -4.0 percentage points $(95 \%$ CI: $-17.1,9.0)$ & $5.6 \%$ decrease & 0.54 \\
\hline
\end{tabular}

Adjusted for the following employer-level variables by IPW estimation: mean employee age, percentage female, racelethnicity (percentage white, Hispanic, black, Asian, or other race), average salary, geographic region, number of employees, percentage of employees with an HDHP, overall generosity of benefits, percentage of employees with each of 15 comorbidities (hypertension, coronary artery disease, congestive heart failure, dementia, schizophrenia, another mental health condition, osteoarthritis, rheumatoid arthritis, non-skin cancer, chronic obstructive pulmonary disease, atrial fibrillation, end-stage renal disease, stroke, peripheral vascular disease, diabetes, or hyperlipidemia), and pre-period employer-level medication adherence 
the patient level, but as a result we were not able to examine the impact of the DHP on individual persons with diabetes who actively participated in the plan. Our estimate of adherence is likely conservative when compared with an estimate limited to persons who took advantage of all DHP features. Second, because we studied the heterogeneous implementation of a health plan as is common in real-world settings, we were not able to elucidate whether employer-level variations in intensity of DHP implementation were associated with differences in predicted adherence. Third, propensity score methods (such as IPW estimation) are unable to control for unmeasured variables at the employer level, such as a "culture of wellness" that may have influenced employer decisions with regard to the DHP. Finally, we did not have information on the details of disease management and wellness programs among the control employers, and were therefore not able to control for these variables in the study analyses. However, the majority of published evidence, including a recent comprehensive review from the RAND Corporation, found that realworld uptake of such programs is very low and that they are not consistently linked to improvements in health or health behaviors. ${ }^{19}$ We do not believe that the presence or absence of such programs will significantly affect our primary outcome of medication adherence.

Our findings add to the evidence of modestly improved adherence linked to reductions in medication cost-sharing for patients, by demonstrating that mean adherence across entire patient populations is enhanced. Linking the incentive of decreased cost-sharing to a requirement that enrollees demonstrate engagement in their own health care, as implemented in the Diabetes Health Plan, is an intriguing option for employers and health plans to consider. The Affordable Care Act stipulates that the Secretary of Health and Human Services develop guidelines for VBID implementation, and additional studies that evaluate different designs as implemented in practice, such as the DHP, will help to inform these critical decisions. ${ }^{20}$

Acknowledgments: This work was presented previously as an abstract at the 36th Annual Meeting of the Society of General Internal Medicine (2013) in Denver, Colorado.

This study was jointly funded by the Centers for Disease Control and Prevention (Division of Diabetes Translation) and the National Institute of Diabetes and Digestive and Kidney Diseases as part of the Natural Experiments for the Translation of Diabetes (NEXT-D) Study (Grant number DP002722).

Dr. Moin received support from the VA Office of Academic Affiliations through the VA Health Services Research and Development Advanced Fellowship Program (TPM65-010), VA Greater Los Angeles Healthcare System, from 2011 to 2014. Dr. Mangione is supported in part by the UCLA Robert Wood Johnson Clinical Scholars Program and the U.S. Department of Veterans Affairs (Grant \#67799). Dr. Mangione also receives support from NIH/NCATS UCLA CTSI Grant Number UL1TROOO124 and from the Barbara A. Levey, M.D., and Gerald S. Levey, M.D., Endowed Chair at the David Geffen School of Medicine at UCLA.

The efforts of Drs. Mangione, Duru, and Steers are supported in part by the University of California, Los Angeles, Resource Center for Minority Aging Research and the Center for Health Improvement of Minority Elderly (RCMAR/CHIME) under NIH/NIA Grant P3OAG021684. Dr. Duru is also supported by NIH Career Development Award K08-AG033360.
The authors would like to thank Ms. Abigail Keckhafer for her assistance in acquiring the UHC data necessary to conduct this study, and Dr. Romain Neugebauer for his helpful guidance on the statistical methods used in this study. The authors would also like to thank Ms. Lindsay Kimbro for her administrative and project management support.

Conflict of Interest: Dr. Ho, Mr. Luchs, Mr. Chan, Ms. Kirvan, and Ms. Keckhafer are employees of UnitedHealthcare. All other authors declare no conflicts.

Corresponding Author: O. Kenrik Duru, MD, MSHS; David Geffen School of Medicine at UCLA, University of California, Los Angeles, CA, USA (e-mail: kduru@mednet.ucla.edu).

\section{REFERENCES}

1. American Diabetes Association. Economic Costs of Diabetes in the U.S. in 2012. Diabetes Care. 2013;36:1033-46.

2. Jha AK, Aubert RE, Yao J, et al. Greater adherence to diabetes drugs is linked to less hospital use and could save nearly $\$ 5$ billion annually. Health Aff (Millwood). 2012;31(8):1836-46.

3. Boswell KA, Cook CL, Burch SP, et al. Associating Medication Adherence With Improved Outcomes: A Systematic Literature Review. Am J Pharm Ben. 2012;4(4):97-108.

4. Ho PM, Bryson CL, Rumsfeld JS. Medication adherence: its importance in cardiovascular outcomes. Circulation. 2009;119(23):3028-35.

5. Weintraub A. Tough love, lower health costs. Available at http://www. bloomberg.com/bw/magazine/content/09_44/b4153056904077.htm. Accessed February 20, 2015.

6. Duru OK, Mangione CM, Chan C, et al. Evaluation of the Diabetes Health Plan to Improve Diabetes Care and Prevention. Prev Chronic Dis. 2013;10:120150.

7. Kimbro LB, Li J, Turk N, et al. Optimizing enrollment in employer health programs: a comparison of enrollment strategies in the Diabetes Health Plan. Am J Manag Care. 2014;20(8):e311-9.

8. Health Savings Accounts and Other Tax-Favored Health Plans. Available at http://www.irs.gov/uac/About-Publication-969. Accessed February 20, 2015.

9. Prasad V, Jena AB. Prespecified falsification end points: can they validate true observational associations? JAMA. 2013;309(3):241-2.

10. Curtis LH, Hammill BG, Eisenstein EL, et al. Using inverse probabilityweighted estimators in comparative effectiveness analyses with observational databases. Med Care. 2007;45(10 Supl 2):S103-7.

11. Rosenbaum PR, Rubin D. The Central Role of the Propensity Score in Observational Studies for Causal Effects. Biometrika. 1983;70(1):4155.

12. Gibson TB, Wang $\mathbf{S}$, Kelly $\mathbf{E}$, et al. A value-based insurance design program at a large company boosted medication adherence for employees with chronic illnesses. Health Aff (Millwood). 2011;30(1):109-17.

13. Gibson TB, Mahoney $\mathbf{J}$, Ranghell $\mathbf{K}$, et al. Value-based insurance plus disease management increased medication use and produced savings. Health Aff (Millwood). 2011;30(1):100-8.

14. Mattke S, Liu H, Caloyeras JP, Huang CY, Van Busum KR, Khodyakov D, Shier V. Workplace Wellness Programs Study. Final Report. Available at http://www.rand.org/content/dam/rand/pubs/research_reports / RR200/RR254/RAND_RR254.sum.pdf Accessed February 20, 2015.

15. Baicker K, Cutler D, Song Z. Workplace Wellness Programs Can Generate Savings. Health Aff. 2010;29(2):304-11.

16. Roebuck CM. Medical Cost Offsets from Prescription Drug Utilization Among Medicare Beneficiaries. J Manag Care Pharm. 2014;20(10):994-5.

17. Carls GS, Roebuck MC, Brennan TA, et al. Impact of medication adherence on absenteeism and short-term disability for five chronic diseases. J Occup Environ Med. 2012;54(7):792-805.

18. Hagen SE, Wright DW, Finch R, et al. Impact of Compliance to Oral Hypoglycemic Agents on Short-term Disability Costs in an Employer Population. Popul Health Manag. 2013

19. Soeren M, Liu H, Caloyeras JP, Huang CY, Van Busum KR, Khodyakov D, Shier V. Workplace Wellness Programs Study Final Report. Santa Monica, Ca: RAND Corporation; 2013.

20. Patient Protection and Affordable Care Act PLN-, §2702, 124 Stat. 119, 318-319, 2010. 